\title{
Gaseous environment in LLAGN: modes of interaction with compact star nuclear population
}

\author{
M. Zajaček ${ }^{1,2}$, V. Karas ${ }^{1}$, L. Šubr ${ }^{2}$, D. Kunneriath ${ }^{1}$ and A. Eckart ${ }^{3,4}$ \\ ${ }^{1}$ Academy of Sciences, Boční II 1401, CZ-14100 Prague, Czech Republic \\ email: michal.zajacek31@gmail.com \\ ${ }^{2}$ Charles University in Prague, V Holešovičkách 2, CZ-18000 Prague, Czech Republic \\ ${ }^{3}$ Universität zu Köln, Zülpicher Strasse 77, D-50937 Köln, Germany \\ ${ }^{4}$ MPIfR, Auf dem Hügel 69, D-53121 Bonn, Germany
}

\begin{abstract}
The Galactic center, which serves as a paradigm of low-luminosity active galactic nuclei (LLAGN), hosts the Nuclear star cluster (NSC) that contains both young and more evolved stars. So far the population of the end-products of stellar evolution has not been observationally confirmed and studied, although there are hints of its presence. We study the distribution of interaction modes of a hypothetical population of neutron stars within the sphere of influence of the Sgr A* supermassive black hole (SMBH). The comparison of our models with future observations could be used to constrain the 3D structure of the Galactic center.
\end{abstract}

\section{Summary of the results}

Interferometric data have been employed to explore the ionized medium near the centre of the Milky Way (Zhao et al. 2010). We use previous results (Kunneriath et al. 2012) about physical conditions of Sgr A West (the Minispiral) to estimate the probability of passages of the putative population of neutron stars of the NSC through the regions of enhanced density, and to assess different modes of the mutual interaction.

Streams of the Minispiral gas are ionized by ultraviolet radiation of massive OB stars present in the NSC. Based on the inferred dynamic mass in the central parsec $\left(\sim 10^{6} M_{\odot}\right)$, it is estimated that at least $\sim 10^{4}$ neutron stars should move in the sphere of influence of the Sgr A* SMBH ( 2pc) (Morris 1993). According to our Monte Carlo simulations, a fraction of this unexplored population $(\lesssim 10 \%)$ propagates through denser ionized medium concentrated along the three arms of the Minispiral. Based on the density and the temperature of the gaseous environment inferred from observations, we analyse interaction regimes of neutron stars passing through this medium. Spectral features are expected to develop within the Minispiral due to non-thermal emission from bow-shocks and pulsar wind nebulae of strongly magnetized stars, and these could be revealed with the improved resolution in the near future (Zajaček 2014a; Zajaček et al. 2014b). The procedure may be applied to other galactic nuclei hosting NSC and the resulting distribution of interaction regimes is expected to vary across different galaxy types.

\section{References}

Kunneriath, D., Eckart, A., Vogel, S. N., et al., 2012, A\& $A$, 538, A127

Morris, M., 1993, ApJ, 408, 496

Zajaček, M., 2014a, MSc. Thesis, Charles University in Prague, Czech Republic

Zajaček, M., Karas, V., Kunneriath, D., 2014b, Proc. of IBWS 2014, Acta Polytechnica, submitted

Zhao, J.-H., Blundell, R., Moran, J. M., et al., 2010, ApJ, 723, 1097 